\title{
LA CONTABILIDAD MEDIOAMBIENTAL: Una fuente inagotable para la investigación universitaria en el Perú
}

\author{
Dr. ERNESTO POLAR FALCÓN
}

En marzo de 1999, las Sociedades Cubanas de Contabilidad, Auditoría, Costos y Finanzas de la Asociación Nacional de Economistas de Cuba realizaron las Primeras Jornadas Científicas: Contabilidad 99 Calidad-Eficiencia-Competitividad, con asistencia de muchos Contadores Públicos y Economistas de América Latina; y de España y Portugal, aportando nuevos temas de discusiones sobre el avance de las ciencias contables en Europa.

Uno de los trabajos que más ha llamado la atención se titula: "Contabilidad Medioambiental: Los Estados Financieros como portadores de Información Medioambiental", presentado por la Universidad de Castilla; La Mancha (España), bajo la firma de tres destacados profesores universitarios: D. Agustín Báidez González, Dr. D. José Antonio Rojas Tercero y Dr. Ángel Tejada Ponce.

El trabajo consta de las cinco siguientes partes:

1. Introducción

2. La Información Medioambiental y la Contabilidad: Contabilidad Medioambiental

3. Información Medioambiental en los

Estados Financieros

3.1 Información a incorporar en el Balance de Situación

3.1.1 Activo

\subsubsection{Pasivo}

3.2 Información a incluir en el Estado de Ganancias y Pérdidas

3.3 Información a incluir en la Memoria 3.4Información Medioambiental a incluir en el Informe de Gestión

3.5 Información a incluir en el Informe de Auditoría

4. Conclusiones

5. Bibliografía-Notas

Al final de este artículo, se presenta integramente este trabajo, materia de análisis y comentarios sobre su contenido. Para hacerlo más comprensible a la lectura de docentes y alumnos, se han cambiado algunos nombres técnicos. Se han anotado aquellos de común uso en la Contabilidad del Perú, en lugar de los que se usan en España, sin modificar en absoluto los conceptos, criterios e ideas que expresan los autores de dicho trabajo profesional.

El Diccionario Larousse dice en los comentarios añadidos a la palabra ambiente, que medio ambiente "es el compendio de valores naturales, sociales y culturales existentes en un lugar y un momento determinados que influye en la vida material y psicológica del hombre". Asimismo, expresa que la ecología "es la parte de la biología que estudia la relación de los seres vivos con la naturaleza" agregando que es "defensa de la naturaleza, protección del medio ambiente". 
También el Diccionario Larousse, cuando describe al Perú, dice entre otras cosas lo siguiente:

- Se distinguen tres cuencas hidrográficas: la del Pacífico con más de treinta ríos de corto curso; la amazónica con los caudalosos ríos principales del Marañón, Ucayali y Amazonas; y la del Lago Titicaca, verdadero mar interior situado a 3815 metros de altitud.

- La línea costeña, de unos $1800 \mathrm{~km}$ de extensión, es recta y poco accidentada, con diversas islas e islotes de superficie reducida, pero con notables depósitos de guano.

- La agricultura es la actividad fundamental del país. En los oasis de la costa, la agricultura es intensiva y de irrigación, se cultiva algodón, caña de azúcar y arroz. En la sierra se produce cereales, patatas y maíz, y en sus valles cálidos, se produce té, café, frutales, etc. De la selva se extraen maderas finas y caucho; y ahora, con gran auge se busca intensamente el petróleo y se explota el que se ubica.

- La ganadería se desarrolla principalmente en la sierra, con mucho ganado vacuno y ovejas. En las partes altas: llamas, alpacas $\mathrm{y}$ vicuñas.

- La pesca está en pleno auge debido a la abundancia de especies marinas en aguas peruanas.

- La minería ocupa un lugar importante en la economía nacional: vanadio (primer productor mundial), bismuto, plata, cobre, oro, estaño, zinc, carbón, fierro, etc.

Frente a esta realidad de recursos naturales que tiene el Perú, el trabajo en mención indica que: "La contabilidad medio- ambiental está referida a la contabilidad de los recursos naturales, en la que se pueden mostrar indicadores, estadísticas e informes sobre consumos, calidades, emisiones, generación de residuos, etc. referidos a áreas geográficas nacionales o regionales". También mencionan lo siguiente:

- Tendría cabida una valorización sobre recursos naturales renovables y no renovables.

- Se contaría con una valiosa información para la toma de decisiones políticas referidas a la economía nacional.

- Los recursos naturales medidos en términos físicos o cuentas físicas, con los cuales se pretende conocer los recursos naturales existentes en un momento determinado de tiempo, identificando para ello los elementos naturales (tierra, aire, agua y paisaje), esta bleciendo unos parámetros de calidad al principio y al final del periodo.

- Establecer la relación entre la actividad económica y el uso de los recursos naturales, en concordancia con las Cuentas Nacionales de la ONU y el cálculo en unidades monetarias, para determinar el gasto interno a la protección del ambiente.

En base a estas explicaciones de medio ambiente, ecología, recursos naturales del Perú y algunos de los conceptos de la contabilidad medioambiental, ¿no debería considerársela como la contabilidad más importante en el Perú, país rico en recursos naturales y con casi nula información contable sobre el uso y reposición de los mismos? ¿No debería contar el Perú con positivas aplicaciones de la contabilidad medioambiental en las empresas dedicadas a la explotación de los recursos naturales y a su inmediata exportación, sin medir las consecuencias ecológicas que ocasionan? Sobre las respuestas a estas preguntas, sería 
conveniente que la profesión contable del Perú tome conciencia del verdadero rol de lacontabilidad medioambiental, y tome como base lo enunciado en el trabajo presentado por la Universidad de Castilla, La Mancha (España).

¿Y la Universidad Peruana, no debería también obligar a todas las Facultades que forman Contadores Públicos a programar cursos de Contabilidad Medioambiental en sus actuales Planes de Estudios, con la finalidad de que las futuras generaciones de Contadores Públicos apliquen las especialidades de la Contabilidad Medioambiental Privada y la Contabilidad Medioambiental Pública, como una obligación moral y profesional importante, en beneficio del Perú, frente a su actual realidad ecológica y la necesidad de preservar sus recursos naturales para el futuro?

Por otro lado, el estudio constante y profundo de las múltiples aplicaciones de la contabilidad medioambiental, deberían ser temas de investigación, tanto para los docentes que enseñan contabilidad en sus diversos niveles, como para los alumnos de pre grado para optar el grado académico de Bachiller en Contabilidad y de los alumnos de post grado, deseosos de optar los grados académicos de Maestría y Doctorado.

Por ejemplo, si en el Punto 3 del trabajo presentado por la Universidad de Castilla, La Mancha (España), titulado: "Información Medioambiental en los Estados Financieros", se buscara interrogantes para desarrollar trabajos de investigación, los conceptos que se indican se pueden convertir en dichos interrogantes, tal como a continuación se precisan:

¿La empresa realiza inversiones en bienes de activo fijo o en gastos de investigación y desarrollo relacionados con el medio ambiente?

- ¿Se han contraído obligaciones para la protección del medio ambiente?

¿En qué costos ha incurrido la empresa, bien en forma obligatoria o voluntaria, que tengan un carácter medioambiental?

- ¿En qué riesgos y contingencias se encuentra envuelta la empresa, derivados de la incidencia de su actividad en el entorno?

¿Qué otras informaciones, bien sean cuantitativas, cualitativas o financieras, se han derivado de la interacción empresa y medio ambiente?

Las dificultades técnicas, que se refieren al problema que supone la evaluación de los costos y beneficios medioambientales, bien podrían transformarse en hipótesis, para aceptarlas o rechazarlas. Lo mismo sucede con las dificultades estratégicas relacionadas con la exteriorización de la información.

En aplicaciones financieras, tanto de las cuentas de activo y pasivo, que se explican en el trabajo, son un reto de constante investigación para estudiar las inversiones fijas, intangibles y el manejo de las existencias que se muestran en el activo, así como los ingresos diferidos y las provisiones que se reflejan en el pasivo, y las acreencias relacionadas con el medio ambiente.

También las partidas de ingresos, costos y gastos, que permiten conocer el comportamiento operativo de una empresa con el medio ambiente y que reflejan el estado de ganancias y pérdidas, son informaciones económicas importantes para la toma de decisiones.

Las inquietudes mostradas en los títulos relacionados con la Memoria, con el Informe 
de Gestión y con el Informe de Auditoría, vinculadas con el medio ambiente, son una novedad para mayores campos de investigación contable y financiera. Asimismo, la bibliografía presentada que permite ampliar el horizonte de la lectura en búsqueda de mayores conocimientos sobre la contabilidad medioambiental. Esto demuestra que el trabajo comentado es un verdadero aporte al desarrollo de la contabilidad en América Latina y, principalmente en el Perú.

El tex to del trabajo presentado por la Universidad de Castilla, La Mancha (España) es el siguiente:

\section{CONTABILIDAD MEDIOAMBIENTAL: Los Estados Financieros como Portadores de Información Medioambiental}

D. Agustín Báidez González Dr. D. José Antonio Rojas Tercero Dr. D. Ángel Tejada Ponce Universidad de Castilla - La Mancha España

\section{INTRODUCCIÓN}

Actualmente el interés y la preocupación por el medio ambiente y su conservación ha pasado a estar contemplado por numerosas ciencias. La mayoría de los estudiosos de las mismas comienzan a plantearse las posibles soluciones y en su caso, qué puede aportar cada una de ellas para evitar el grave deterioro medioambiental que está sufriendo nuestro entorno.

El término verde (para hacer referencia al medio ambiente), se está aplicando constantemente a productos e ideas que tienen alguna relación con el mismo, así, se habla de productos verdes, publicidad verde, economía medioambiental, información medioambiental, $y$ en nuestro caso, conta- bilidad medioambiental. Creemos que esta ciencia podrá también aportar su grano de arena a la conservación y protección de nuestro medio ambiente. Esta última cuestión es la que vamos a tratar de aclarar en nuestro pequeño trabajo.

\section{LAINFORMACIÓN MEDIOAMBIENTAL Y LA CONTABILIDAD: CONTABILIDAD MEDIOAMBIENTAL}

El término contabilidad medioambiental puede tener varios significados, dependiendo al campo contable que se relacione. Podemos asociar el referido término a tres contextos contables:

a) Contabilidad medioambiental en el contexto de la contabilidad nacional, referida a la contabilidad de los recursos naturales, en la cual se pueden mostrar indicadores, estadísticas e informes sobre consumos, calidades, emisiones, generación de residuos, etc. referidos a áreas geográficas nacionales o regionales.

También tendría cabida una valoración sobre recursos naturales renovables y no renovables. La contabilidad nacional, por tanto, contaría con una valiosa información para la toma de decisiones políticas referidas a la economía nacional. Estas decisiones podrán contribuir a la conservación del medio, si cuando se adoptan, se cuenta con la información medioambiental correspondiente. Todo ello supone incorporar en la propia contabilidad nacional el aspecto medioambiental como una variable más.

Las distintas alternativas para introducir información medioambiental en la contabilidad nacional hacen referencia a:

1. Los recursos naturales medidos en términos físicos o cuentas físicas. Con 
el uso de estas cuentas se pretende conocer qué recursos naturales existen en un momento determinado de tiempo, identificando para ello los elementos naturales (tierra, aire, agua y paisaje), estableciendo unos parámetros de calidad al principio y al final del periodo.

2. Cuentas satélites. Se intenta establecer una relación entre la actividad económica y el uso de los recursos naturales, siguiendo para ello los principios y reglas establecidos en el Sistema de Cuentas Nacionales de la ONU, obteniendo información económica sobre el medio ambiente y calculando, en unidades monetarias, el gasto interno dedicado a la protección del ambiente.

3. La modificación o elaboración de nuevos indicadores económicos que permitan suministrar información medioambiental a nivel macroeconómico.

La contabilidad medioambiental en el contexto de la contabilidad nacional va a tratar no sólo de asignar valores monetarios a los impactos ambientales, o al agotamiento y la degradación de los recursos derivados de la actividad económica, sino que a través de ella se va a conocer el patrimonio ambiental como un stock, así como su posible evolución, de ahí la necesidad de contar con un Inventario o estado ambiental que oriente a las Administraciones Públicas e informe a los usuarios en general.

b) Contabilidad medioambiental como un aspecto de la contabilidad de gestión, contribuyendo a la gestión de las empresas a la hora de determinar costos medioambientales, evaluar proyectos sobre inversiones de capital con carácter medioambiental, y ayudar en la toma de decisiones en la misma, dada la creciente interacción empresa-medioambiente. c) Contabilidad medioambiental en el contexto de la contabilidad financiera, estando ésta referida a la preparación de los estados financieros para los usuarios extemos, usando los principios contables generalmente aceptados. La contabilidad financiera en este contexto, tendrá el papel de estimar y publicar información referida a costos medioambientales, pasivos medioambientales, contingencias medioambientales, etc., y cuanta otra información de carácter medioambiental tenga cabida en los mismos.

Los sistemas contables en sus distintos contextos, consideramos que van a ser capaces de presentar información medioambiental de forma adecuada y en tiempo oportuno, sobre datos relevantes derivados de acciones o hechos que hayan afectado, afecten o puedan afectar en un futuro al medio ambiente.

Esta comunicación se centrará en el estudio de la presentación de información de carácter medioambiental en los estados financieros externos presentados por las empresas, de acuerdo con los principios y normas contables, generalmente aceptados y en base a la estructura tradicional de los mismos. El objetivo inicial de los estados financieros tradicionales, es el de comunicar información económico-financiera relativa al patrimonio de la empresa en un momento determinado, así como la referida a los resultados del ejercicio. Creemos que este objetivo puede llegar mucho más allá, incorporando información medioambiental, tanto la derivada de acciones o medidas que hayan afectado al patrimonio empresarial en el pasado, como de aquellas otras que puedan afectar en un futuro. Además, también se considerará interesante incluir en los referidos estados, información medioambiental relacionada con la interacción empresa-medio ambiente, aunque no haya 
afectado ni vaya a afectar en el patrimonio empresarial, enriqueciendo de esta manera el contenido medioambiental de estos documentos contables.

La creciente demanda de información medioambiental por parte de los distintos usuarios de la contabilidad financiera, va a suponer que la información financiera externa se esté encaminando a responder a las necesidades comunicacionales entre los distintos sujetos demandantes de información económica, lo que supone un cambio en la concepción de la contabilidad como mero sistema representativo de la información económica, pasando a ser contemplada como sistema de comunicación entre las distintas personas implicadas en el proceso económico.

\section{INFORMACIÓN MEDIOAMBIENTAL EN LOS ESTADOS FINANCIEROS}

La información medioambiental a divulgar por los estados financieros podría abarcar los siguientes conceptos:

a) Inversiones que se realicen ya sea bien en bienes de activo fijo o gastos de investigación y desarrollo relacionados con el medioambiente.

b) Obligaciones contraídas para la protección del medio ambiente.

c) Costos en que incurra la empresa, bien de forma obligatoria o voluntaria, y que tengan un carácter medioambiental.

d) Riesgos y contingencias en que la em presa se encuentre envuelta, derivados de la incidencia de su actividad en el entomo.

e) Cualquier otra información derivada de la interacción empresa o medio ambiente, bien sea cuantitativa, cualitativa o financiera.

La exteriorización de la información medioambiental se va a encontrar con numerosas dificultades. Christophe establece dos tipos de dificultades a la hora de implantar un sistema de información medioambiental,dificultades técnicas y dificultades estratégicas.

Las dificultades técnicas se refieren al problema que supone la evaluación de los costos y beneficios medioambientales:

1) Excesiva complejidad técnica en los procesos medioambientales, que requiere un estudio por parte de técnicos de varias disciplinas. Esta circunstancia supone una complicada asignación de costos entre los diferentes programas ambientales.

2) La actualización de valores futuros está muy influenciada por variables poco previsibles, por ejemplo, tecnología, precios, escenarios políticos, etc.

3) Los costos reales son muy diferentes de los que soporta la empresa.

4) Existen costos difícilmente controlables por la empresa, como la evolución de los productos en manos de los consumidores.

Dificultades estratégicas relacionadas con la exteriorización de la información.

1) Tentación por parte de la gerencia para eliminar las malas noticias, informando solamente las que le interesan.

2) Ausencia de metodologías que permitan la comparación de las realizaciones en el tiempo y en el espacio.

3) Inutilidad aparente de la información me dioambiental. 
4) La publicación de información sobre aspectos ambientales pueden considerarse por parte de la gerencia como mala imagen pública, o pistas para las autoridades que pueden sancionar a la empresa.

La distinta información medioambiental que sea objeto de publicación tendrá que estar ubicada en los distintos documentos que utiliza el modelo contable tradicional. Así distinguiremos la información medioambiental a incorporar en:

- Balance de Situación.

- Estado de Ganancias y Pérdidas o Estado de resultados.

- Memoria.

- Informe de Gestión.

- Informe de Auditoría.

\subsection{Información a incorporar en el} balance de situación.- El balance como estado contable estático, va a mostrar la realidad económico-financiera de la empresa en un momento determinado.

El balance de situación muestra la imagen fiel del patrimonio y la situación económico-financiera de la empresa en un momento dado, recogiendo por un lado, la materialización de los recursos obtenidos por la empresa (activo) y por otro, el origen de dichos recursos (pasivo).

Rivero Torres afirma que el balance completo debe informar no sólo de la situación actual estricta del patrimonio, sino también sobre los demás aspectos complementarios que ayudan a obtener una visión total de las circunstancias en que está inmerso y que pueden alterarlo en el futuro.

Se puede interpretar que entre las circunstancias en que está inmerso el patrimonio y que pueden alterarlo en el futuro, se encuentran cuantas eventuali- dades y contingencias puedan afectar al patrimonio de la empresa. Por tanto, y en vista de la interpretación anterior, tendría cabida en el balance, la información de carácter medioambiental que se derivase de circunstancias que pudiesen afectar al patrimonio empresarial en el presente o en el futuro.

Serán objeto de análisis las partidas del balance de situación y del estado de ganancias y pérdidas, que pueden tener una incidencia medioambiental, siendo conveniente la distinción a través de subcuentas para poder identificarlas de una mejor forma.

3.1.1 Activo.- En cuanto al activo fijo habrá que contabilizar por separado los terrenos y construcciones que se encuentren afectados por la contaminación. Sobre dichos activos deberán realizarse las correspondiente correcciones valorativas por pérdidas de valor que pudieran sufrir.

También habrá que reflejar por separado, toda la maquinaria, instalaciones y utillaje, que por razones medioambientales (como por ejemplo adaptación a una nueva normativa) deban ser sustituidas o reformadas. En otras ocasiones dichos activos sufrirán un desgaste y obsolescencia superior al normal debido al propio proceso de producción.

No debemos olvidar la realización de inversiones en activos materiales por razones exclusivamente medioambientales, como pueden ser la adquisición de filtros, depuradoras, etc. Todas ellas deberían ser identificadas de forma separada en el balance de situación.

En lo referente a los activos intangibles, es posible que la empresa lleve a cabo proyectos de investigación y desarrollo relacionados con el medio ambiente. En ocasiones serán proyectos encaminados a 
desarrollar nuevas tecnologías para eliminar residuos o vertidos ya producidos, y en otras, la investigación estará encaminada a la reducción de la emisión y generación de los mismos.

También se pueden realizar inversiones en programas informativos para el control medioambiental en la empresa, que serán objeto de una identificación específica en el balance de situación.

Los gastos a distribuir en varios ejercicios con carácter medioambiental deberán ser identificados de forma separada. Tendrán cabida en esta partida los gastos o costos medioambientales que sean objeto de capitalización debido a su incidencia a lo largo de varios ejercicios.

El activo corriente de la empresa puede estar compuesto por existencias de materias primas o productos que sea conveniente su identificación como productos con incidencia medioambiental, bien por que son contaminantes, bien por que su no adecuada manipulación puede ocasionar desastres ecológicos o bien porque son ecológicos; es decir, que están fabricados para que no degraden el medio ambiente.

Tienen una importancia especial, las partidas de residuos y desechos que puedan derivarse de los procesos productivos, y que sean contaminantes. Una identificación de los mismos en las cuentas anuales permitiría un análisis exhaustivo de la empresa en materia de generación de residuos.

Merecen atención especial los productos reciclables, que habrán de tener una identificación especial.

Será conveniente reflejar de forma separada todas las provisiones sobre activos medioambientales que se hayan dotado como consecuencia de la pérdida de valor de las distintas categorías de activos, siempre y cuando tengan el carácter de reversibles.

3.1.2 Pasivo.- En el pasivo del balance cabe destacar los ingresos a distribuir en varios ejercicios, donde se encuadrarán las subvenciones relacionadas con el medio ambiente, que cada vez son más frecuentes.

En cuanto a las provisiones para riesgos y gastos, se reflejarán todas las provisiones por responsabilidades medioambientales. Estas provisiones estarán diferenciadas del resto, de forma que se pueda identificar el riesgo y contingencias en que se puede encontrar envuelta la empresa.

La masa patrimonial de acreedores reflejará también todas aquellas deudas y obligaciones contraídas y relacionadas con el medio ambiente.

\subsection{Información a incluir en el estado de ganancias y pérdidas.- El estado de} ganancias y pérdidas como estado contable dinámico muestra el resultado de un ejercicio, así como los componentes que lo han generado. Este estado contable comprende con la debida separación, los ingresos y los gastos del ejercicio y, por diferencia, el resultado del mismo.

En el estado de ganancias y pérdidas debe quedar reflejado anualmente los gastos e ingresos de carácter medioambientales que se devengan. Se utilizarán una serie de subcuentas específicas donde queden reflejados dichos gastos e ingresos, y de esta forma poder conocer en qué medida las variables medioambientales influyen en el resultado de la empresa. Tras un análisis de la información medioambiental que presenta el estado de ganancias y pérdidas, también podremos observar el comportamiento de la empresa en materia de medio ambiente. 
En el capítulo de gastos, la empresa deberá identificar todos los consumos de materiales, costos de mano de obra, servicios, amortizaciones, etc. que se encuentren relacionados con el medio ambiente.

Algunos grupos de costos medioambientales que pueden reflejarse en la Contabilidad de modo diferenciado serían:

- Control de costos de fugas y derrames (materias, deterioro de producción, limpieza, residuos generados).

- Costos de mantenimiento (Inspección, limpieza, lubricación, comprobación, reemplazo de piezas) de instalaciones medioambientales.

- Costo de gestión de residuos generados, emisión y vertido (producción, transporte, almacenamiento, manipulación).

- Costo de gestión de inversiones relacionadas con el medio ambiente, depuradoras de agua, sistemas de reciclado, recuperación, ruidos, olores, emisiones, etc.

- Partidas de gastos (no activables), propiedad industrial, etc.

- Dotación al fondo de reversión.

- Dotación a la amortización y provisiones, relacionadas con partidas del balance calificadas medioambientales.

Otros costos varios relacionados con la actuación medioambiental, y que también deben identificarse en el estado de ganancias y pérdidas son las siguientes:

- Cánones, seguros medioambientales, autorizaciones, licencias y permisos, derechos por uso de tecnología externa, tarifas de vertederos, etc.

- Tributos ecológicos, multas y sanciones administrativas y penales.

- Manipulación de contenedores y envases retornables.

- Almacenamientos especiales.
- Pérdidas de materiales en pruebas por obsoletos.

- Análisis de vida de productos.

- Evaluaciones de impacto ambiental.

- Planes de emergencia.

- Sistemas de información a la dirección para la gestión medioambiental.

- Gasto por embellecimiento medioambiental de la ubicación.

- Publicidad de nuevos ecoproductos.

- Gastos de información y formación medioambiental.

- Gastos de equipamientos menores y elementos consumibles de análisis laboratorios, etc.

- Gastos de expertos para realizar auditorías, evaluaciones y peritaciones.

- Análisis y laboratorios externos.

- Costo de creación y mantenimiento de mercados ecológicos.

Entre las partidas de ingresos que la empresa debe identificar de forma separada encontramos las siguientes:

- Ingresos por venta o alquiler de tecnologías relacionadas con el medio ambiente.

- Subvenciones de explotación recibidas por la empresa por razones medioambientales.

- Imputación al ejercicio de subvenciones de capital relacionadas con el medio ambiente.

\subsection{Información a incluir en la memo-} ria.- La memoria completará, ampliará y comentará el balance y el estado de ganancias y pérdidas. Será conveniente por tanto, que la memoria complete y comente la información medioambiental que se encuentra en el balance y en el estado de ganancias y pérdidas, así como, que incluya aquella otra información con trascendencia medioambiental que no se haya podido incluir en los estados anteriores. 
El Centro de Empresas Multinacionales, de la ONU, estableció una serie de recomendaciones sobre la información que deberían suministrar las empresas en la memoria de las cuentas anuales. En lo referente a los criterios contables se debería de explicar lo siguiente:

a) La dotación de gastos o, en su caso, el criterio de capitalización y las políticas de amortización.

b) La dotación de provisiones o la creación de reservas para atender desastres ecológicos.

c) La divulgación de la información sobre los pasivos eventuales.

Conjuntamente con los criterios contables debería incluirse en la memoria información sobre los importes referentes a estas partidas.

Otra información que debería recoger sería la referente a:

a) Programas medioambientales llevados a cabo por la empresas.

b) Informes sobre el resultado de auditorias medioambientales realizadas.

c) Informes sobre el cumplimiento de la normativa medioambiental vigente.

d) Cualquier otra información que tuviese una trascendencia medioambiental.

\subsection{Información medioambiental a in-} cluir en el informe de gestión.- El informe de gestión es un documento contable que debe de acompañar a las cuentas anuales (balance, estado de ganancias y pérdidas y memoria). Sería conveniente incluir en este informe, un apartado adicional en el que se hiciera referencia a la actitud que la empresa tiene con el medio ambiente.

El Centro de Empresas Multinacio- nales de la ONU recomienda que en el informe de gestión se incluya la siguiente información:

a) Cuestiones medioambientales que afectan a la empresa y a sus sectores.

b) La política y los programas oficiales que la empresa ha aprobado respecto a medidas de protección ambiental.

c) Las mejoras que se han logrado desde la introducción de la política o aprobación del programa (o desde los cinco últimos años si este periodo fuera menor).

d) Los objetivos que la empresa se haya fijado respecto a las emanaciones que afectan al medio ambiente y los resultados que la empresa ha logrado en el cumplimiento de esos objetivos.

e) El grado de aplicación de las medidas de protección medio ambiental impuestas por la legislación estatal y, en tal caso, en qué medidas se han cumplido los requisitos legales (por ejemplo, para la reducción de las emanaciones).

f) Prácticas materiales establecidas en las leyes sobre el medio ambiente.

g) Consecuencias financieras de las medidas de protección ambiental en relación con las inversiones y con los ingresos de la sociedad, tanto para el ejercicio en curso como para ejercicios futuros.

h) Desglose de los gastos de explotación con una descripción de las medidas de protección ambiental con las que se relacionan. Este desglose debería incluir las siguientes partidas:

1.- Tratamiento de afluentes líquidos. 
2.- Tratamiento del aire y de los gases desechos.

3.- Tratamiento de desechos sólidos.

4.- Análisis, control y cumplimiento.

5.- Medidas correctivas.

6.- Reciclaje.

7.- Otras partidas.

En caso de que esta información no se hubiera podido desglosar, se deberían de dar las razones que lo impidieran.

i) Importe de los gastos capitalizados en el ejercicio, así como los plazos de amortización previstos.Estas partidas deberían desglosarse en la medida de lo posible de la forma señalada para los gastos de explotación.

\subsection{Información a incluir en el informe de auditoría - La información que contienen las cuentas anuales en muchos casos debe ser verificada por parte de los auditores externos. El área medioambiental también será objeto de atención por parte de estos técnicos a la hora de emitir su opinión en el informe de auditoría.}

La actuación de los auditores en este caso, estará encaminada a comprobar aquellos aspectos medioambientales mostrados en los distintos estados financieros.

Entre los aspectos que más deben de preocupar a los auditores cuando se disponen a auditar el área medioambiental cabría mencionar.

1. La posibilidad de que las empresas ocultan o valoren a la baja las provisiones y contingencias de carácter medioambiental, (obligaciones derivadas de operaciones de limpieza u otras responsabilidades incurridas por el deterioro causado en el entorno).
2. El hecho de que el tratamiento y presentación dados en los estados financieros no hubiese sido el adecuado.

En caso de que los auditores lo consideren oportuno, podrán poner de manifiesto en el informe de auditoría aquellas salvedades relativas a la información medioambiental que presentan los estados financieros, e incluso en algunos casos, cuando la importancia lo determine, podrán llegar a emitir una opinión desfavorable.

\section{CONCLUSIONES}

Los problemas medioambientales derivados de la relación empresa medio ambiente, son cada vez más frecuentes y de mayores dimensiones. Esto, unido a la creciente demanda de los usuarios de la información contable, sobre aspectos sociales y medioambientales relacionados con la empresa y con el entorno, va a suponer la necesidad para la misma de informar a terceros sobre la incidencia que su actividad provoca sobre éste, y sobre la actitud adoptada por la empresa para evitar, reducir o en su caso reparar los daños causados.

La contabilidad financiera, a través de sus estados informativos (balance, estado de ganancias y pérdidas, memoria...), va a ser capaz de exteriorizar información medioambiental en sus distintas vertientes: información financiera, información cuantitativa e información cualitativa, todo ella dirigida a los usuarios externos, de forma que éstos pueden observar y en su caso analizar el patrimonio y los resultados de la empresa, contemplando las variables medioambientales, todo ello, en aras de obtener una imagen fiel de los mismos

No obstante, somos conscientes de las distintas dificultades que se pueden presentar 
para incorporar en los estados financieros algunas categorías de información medioambiental, sobre todo a la hora de su medición y cuantificación. A pesar de ello, creemos que toda la información medioambiental puede tener cabida en los referidos estados. Si se trata de información financiera se podrá incorporar en el balance o en el estado de ganancias y pérdidas, y en su caso en la memoria. Cuando lo que se trate sea de información cuantitativa o cualitativa será objeto su incorporación en la memoria, en el informe de gestión, y en su caso, los auditores externos la podrán incorporar en el informe de auditoría.

\section{BIBLIOGRAFÍA}

CARMONA IBÁNEZ, P.

"La información medioambiental. Contabilidad y auditoría" . Partida Doble $\mathrm{N}^{\circ}$ 76, marzo 1997.

ELORRIAGA ANCIN, J. F.

"El coste de la operatividad de la gestión medioambiental de la empresa". Jomadas de Contabilidad de Gestión. Alcalá de Henares, p. 266.1993.

ENVIRONMENTAL PROTECTION AGENCY'S. "An introduction to environmental accounting as a business tool: Key concepts and terms".

GRUPO INTERGUBERNAMENTAL DETRABAJO DEEXPERTOS EN NORMAS INTERNACIONALES DECONTABILIDAD Y PRESENTACIÓN DEINFORMES.

"Conclusión sobre la contabilidad y la presentación de informes en las empresas transnacionales", Naciones Unidas, Nueva York y Ginebra, 1994.

LÓPEZRODRÍGUEZ,R.

"Armonización de la información sobre las medidas financieras. Trabajos realizados por el I.S.A.R. " En: Revista Española de Financiación y Contabilidad, Volumen XXII. Número 75.

MUÑOZRODRÍGUEZ,C.

"Los sistemas contables y el medio ambiente". Partida Doble No 76, marzo, 1997.

QUESADA SÁNCHEZ.F.J.

"Análisis del patrimonio de la empresa". Edición del autor. Impreso en Tebar Flores, Madrid, 1993.

RIVEROTORRE,P

"Análisis de balances y estados complementarios".Editorial Pirámide.Tercera edición, Madrid, 1988.

\section{NOTAS}

ENVIRONMENTAL PROTECTION AGENCY'S. "An Introduction to environmental accounting as a business tool: Key concepts and Terms". p. 28 y ss.

Un análisis más exhaustivo de estas alternativas puede verse en MUÑOZ RODRÍGUEZ, C. "Los sistemas contables y el medio ambiente". Partida Doble $\mathrm{N}^{\circ} 76$, marzo 1997, p.36 y ss.

\section{MUÑOZRODRÍGUEZ,C.}

"Los sistemas contables y el medio ambiente" Partida doble $\mathrm{N}^{\mathbf{0}} 76$ - marzo 1997.- p. 36.

QUESADA SÁNCHEZ,F. J.

Presentación de la información anual. Edición del autor. Impreso en Tebar Flores, Madrid ,1994- p. 19

BAIDEZ GONZALES, A. y NEVADO PEÑA, D."Información social medioambiental: Un objetivo a conseguir por las empresas". VI Encuentro de Profesores Universitarios de 
Contabilidad.Madrid 26, 27 y 28 de mayo de 1994.

CHRISTOPHE B.

"Comptabilité et Environment. Prise en Compte des Activités environnementales dans les Documents Financiers des Entreprises". Tesis de Doctorado, 1989.

QUESADA SÁNCHEZ,F. J.

"Análisis del patrimonio de la Empresa". Edición del autor. Impreso en Tebar Flores, Madrid, 1993.

RIVEROTORRE.P. A.

"Análisis de balances y estados complementarios". Editorial Pirámide. Tercera edición, Madrid ,1988 , p. 42

ELORRIAGA ANCIN,J.F.

"El coste de la opertividad de la gestión medioambiental de la empresa". Jomadas de Contabilidad de Gestión. Alcalá de Henares 1993. p. 266

ELORRIAGA ANCIN, J.F., op. cit. p. 266

LOPEZRODRÍGUEZ, R., op. cit. p.341

GRUPO INTERGUBERNAMENTAL DE TRABAJO DE EXPERTOS EN NORMAS INTERNACIONALES DECONTABILIDAD Y PRESENTACIÓN DE INFORMES.

"Conclusión sobre la contabilidad y la presentación de informes en las empresas transnacionales".Naciones Unidas. Nueva York y Ginebra,1994, p. 24

CARMONA IBÁÑEZ,P.

"La información medioambiental. Contabilidad y auditoría". Partida doble $\mathrm{N}^{\circ} 76$. marzo 1997, p. 45 